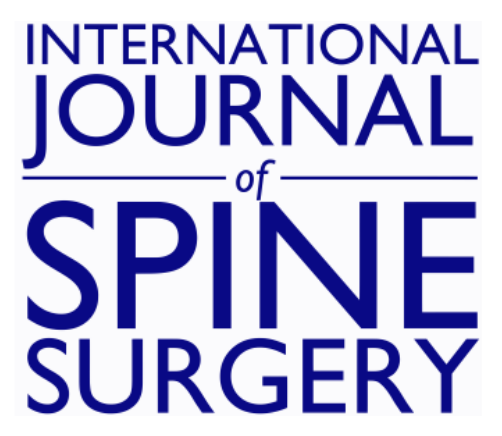

\title{
Risk Factors for Postoperative Urinary Tract Infections Following Anterior Lumbar Interbody Fusion
}

DANNY LEE, RYAN LEE, MEGAN T. CROSS, UCHECHI IWEALA, JEFFREY H. WEINREB, DAVID P. FALK, JOSEPH R. O'BRIEN and WARREN YU

Int J Spine Surg 2020, 14 (4) 493-501

doi: https://doi.org/10.14444/7065

http://ijssurgery.com/content/14/4/493

This information is current as of April 26, 2023.

Email Alerts Receive free email-alerts when new articles cite this article. Sign up at: http://ijssurgery.com/alerts 


\title{
Risk Factors for Postoperative Urinary Tract Infections Following Anterior Lumbar Interbody Fusion
}

\author{
DANNY LEE, MD,${ }^{1}$ RYAN LEE, MBA, ${ }^{1}$ MEGAN T. CROSS, MD, ${ }^{1}$ UCHECHI IWEALA, MD, MBA, ${ }^{2}$ \\ JEFFREY H. WEINREB, MD, ${ }^{2}$ DAVID P. FALK, MD ${ }^{3}$ JOSEPH R. O'BRIEN, MD, MPH, ${ }^{4}$ \\ WARREN YU, MD ${ }^{2}$ \\ ${ }^{1}$ The George Washington University School of Medicine and Health Sciences, The George Washington University, Washington, D.C., ${ }^{2}$ Department of Orthopaedic \\ Surgery, The George Washington University, Washington, D.C., ${ }^{3}$ Department of Orthopaedic Surgery, The University of Pennsylvania, Philadelphia, \\ Pennsylvania, ${ }^{4}$ Washington Spine and Scoliosis Clinic, OrthoBethesda, Bethesda, Maryland.
}

\begin{abstract}
Background: Although risk factors contributing to UTI have been studied in posterior approaches to lumbar fusion, there is a lack of literature on factors contributing to UTI in anterior lumbar interbody fusion (ALIF). Our purpose was to identify preoperative independent risk factors for postoperative urinary tract infection (UTI) following anterior lumbar interbody fusion (ALIF) so that surgeons may be able to initiate preventative measures and minimize the risk of UTI-related morbidity following ALIF.

Methods: The American College of Surgeons-National Surgical Quality Improvement Program database was queried to identify 10232 patients who had undergone ALIF from 2005 to 2016; 144 patients (1.41\%) developed a postoperative UTI while 10088 patients $(98.59 \%$ ) did not. Univariate analyses were conducted to compare the 2 cohorts' demographics and preoperative comorbidities. Multivariate logistic regression models were then utilized to identify significant predictors of postoperative UTI following ALIF while controlling for differences seen in univariate analyses.

Results: Age $\geq 60$ years $(P=.022)$, female sex $(P<.001)$, alcohol use $(P=.014)$, open wound or wound infections $(P=.019)$, and steroid use $(P=.046)$ were independent risk factors for postoperative UTI. Longer operative times were also independent predictors for developing UTI: 120 minutes $\leq \mathrm{x}<180$ minutes $(P=.050), 180$ minutes $\leq \mathrm{x}<240$ minutes $(P=.025)$, and $\geq 240$ minutes $(P=.001)$. Postoperative UTI independently increased the risk for pneumonia, blood transfusions, sepsis, thromboembolic events, and extended length of stay as well.

Conclusions: Age $\geq 60$ years, female sex, alcohol use, steroid use, and open wound or wound infections independently increased the risk for UTI following ALIF. Future work analyzing the efficacy of tapering alcohol and steroid use preoperatively and reducing procedural time with the aim of lowering UTI risk is warranted. Preoperative wound care is strongly encouraged to decrease UTI risk.
\end{abstract}

Level of Evidence: III.

Lumbar Spine

Keywords: anterior lumbar interbody fusion, urinary tract infection, anterior approach, operative time

\section{INTRODUCTION}

Urinary tract infections (UTIs) are a common example of nosocomial infections in the postoperative period. Indwelling catheter use increases the risk for UTIs as the artificial plastic surface serves as a nidus for multiple species of bacterial growth that can lead to cystitis, and in some cases, pyelonephritis. ${ }^{1,2}$ Accounting for almost $40 \%$ of all nosocomial infections, UTIs contribute significantly to increased length of stay, mortality risk, sepsis, superficial infection, and the economic burden following hospitalizations. $^{3-6}$ In the United States, many insurers have deemed nosocomial UTIs as preventable adverse events for which reimbursement will adversely affected, so there is an increasing financial pressure for surgeons and institutions alike to decrease the incidence of UTIs as well. ${ }^{7}$

Anterior lumbar interbody fusion (ALIF) has been a mainstay treatment modality for a variety of conditions affecting the lumbar spine, including degenerative disc disease, pseudarthrosis, and spondylolisthesis. ${ }^{8-10}$ ALIF has been proposed to have several biomechanical advantages when compared to posterior approaches including preservation of posterior spinal musculature, more direct visualization of the disc space, and decreased development of adjacent segment disease. ${ }^{1-13}$ Although risk factors contributing to UTI have been studied in posterior approaches to lumbar fusion, there is a lack of 
literature on factors contributing to UTI in ALIF. ${ }^{14}$ This current study seeks to establish risk factors that predispose patients undergoing ALIF to developing UTIs by utilizing The American College of Surgeons-National Surgical Quality Improvement Program (ASC-NSQIP) database so that surgeons can better stratify patients and take appropriate prophylactic measures to prevent certain postoperative complications that are associated with UTIs.

\section{METHODS}

The ACS maintains a multi-institutional clinical registry of over 5,000,000 surgical patients who have undergone procedures from 2005 to 2016 in its NSQIP database. The ACS-NSQIP database contains 274 variables for each surgical patient detailing demographics, operative parameters, and postoperative complications.

\section{Patient Cohort Selection}

The ACS-NSQIP was queried to identify patients who had undergone ALIF from 2005 to 2016. Patients with Current Procedure Terminology (CPT) codes 22558 and 22585 were isolated for further analysis. 10232 patients who had undergone ALIF were included in this retrospective study. It is important to note that 22558 also refers to lateral approaches to the spine (extreme lateral interbody fusion, lateral lumbar interbody fusion, and oblique lateral lumbar interbody) at the time of writing of this paper. All included patients were $\geq 18$ years old and had complete information regarding the incidence of postoperative UTIs following their ALIF procedures. Patients were then stratified into 2 separate cohorts - those who had experienced postoperative UTIs and those who did not. These 2 patient cohorts were then analyzed for differences in demographics, preoperative comorbidities, and additional complications.

\section{Variables}

The demographic factors included in this retrospective study were age, sex, race, and body mass index. Only preoperative comorbidities that had more than an $85 \%$ response rate were included for analysis in this study. The list of complete comorbidities and administrative or operative variables included in this study can be found in Tables 1 and 2, respectively. ${ }^{15,16}$ Demographic factors and operation-specific parameters were analyzed for differ- ences between the 2 patient cohorts to identify risk factors for UTIs following ALIF. Incidence of additional postoperative complications were also analyzed for differences between the 2 cohorts in the 30 -day period following their procedures. The complete list of postoperative complications included in this study can be found in Table 3 .

\section{Statistical Analysis}

Univariate analyses were used to assess differences in the incidence of patient demographic factors, preoperative comorbidities, operation-specific variables, and postoperative complications between the 2 cohorts. Pearson $\chi^{2}$ tests and Fischer exact tests (expected cell sizes $<5$ ) were utilized to evaluate differences in prevalence rates of categorical variables expressed as number and rate of incidence. One-way analyses of variance were utilized for continuous variables, such as age, laboratory values, and time variables. These variables were expressed as mean values with respective standard deviations. All statistical findings for univariate analyses with $P$ values less than or equal to .05 were considered significant for this retrospective study.

Variables that were found to be significantly different in prevalence upon univariate analyses were included in the multivariate logistic regression models in order to identify significant risk factors for postoperative UTIs in patients undergoing ALIF.

Multivariate logistic regression models were developed to identify complications that may be associated with UTIs following ALIF by controlling for significant demographic factors and preoperative comorbidities. All statistical findings with $P$ values less than or equal to .05 in the multivariate analyses were considered significant for this retrospective study. All statistical analyses were completed using the IBM SPSS Statistics Version 25 software (IBM Corporation, Armonk, New York).

\section{RESULTS}

By querying the ACS-NSQIP database, we identified 10232 patients who had undergone anterior approaches to the spine from 2005 to 2016, of whom $144(1.41 \%)$ had developed postoperative UTI and 10088 whom $(98.59 \%)$ had not. These 2 cohorts of patients were analyzed for differences in various characteristics; those who had developed postoperative UTIs were significantly older (61.67 years, SD 13.283 versus 55.27 years, 
Table 1. Demographics and comorbidities stratified by postoperative UTI.

\begin{tabular}{|c|c|c|c|c|c|}
\hline & \multicolumn{2}{|c|}{ Control } & \multicolumn{2}{|c|}{ UTI } & \multirow[b]{2}{*}{$P$ value } \\
\hline & No. & $\%$ & No. & $\%$ & \\
\hline \multicolumn{6}{|c|}{ Demographics } \\
\hline Total & 10088 & & 144 & & \\
\hline Age $(\text { mean } \pm \mathrm{SD})^{\mathrm{b}}$ & $55.27 \pm 13.627$ & & $61.67 \pm 13.283$ & & $<.001$ \\
\hline Sex & & & & & $<.001$ \\
\hline Female & 5384 & 53.37 & 112 & 77.78 & \\
\hline Male & 4702 & 46.61 & 32 & 22.22 & \\
\hline Race & & & & & .875 \\
\hline American Indian or Alaska Native & 44 & 44 & 0 & 0.00 & \\
\hline Asian or Pacific Islander & 137 & 1.36 & 1 & 0.69 & \\
\hline Black or African American & 735 & 7.29 & 10 & 6.94 & \\
\hline Hispanic & 5 & 0.05 & 0 & 0.00 & \\
\hline White & 8618 & 85.43 & 125 & 86.81 & \\
\hline Body mass index $\left(\mathrm{kg} / \mathrm{m}^{2}\right)$ & & & & & .055 \\
\hline Normal $(<25.0)$ & 2101 & 20.83 & 30 & 20.83 & \\
\hline Overweight (25.0-29.9) & 3390 & 33.60 & 46 & 31.94 & \\
\hline Class I (30.0-34.9) & 2668 & 26.45 & 31 & 21.53 & \\
\hline Class II (35.0-39.9) & 1289 & 12.78 & 20 & 13.89 & \\
\hline Class III $(>40.0)$ & 609 & 6.04 & 17 & 11.81 & \\
\hline \multicolumn{6}{|c|}{ Preoperative comorbidities $^{c}$} \\
\hline Obesity (Class I, II, and III) & 4566 & 45.26 & 68 & 47.22 & .663 \\
\hline Diabetes mellitus & & & & & .013 \\
\hline No diabetes mellitus & 423 & 4.19 & 12 & 8.33 & \\
\hline Non-insulin dependent & 906 & 8.98 & 18 & 12.50 & \\
\hline Insulin dependent & 8759 & 86.83 & 114 & 79.17 & \\
\hline Smoke & 2359 & 23.38 & 20 & 13.89 & .007 \\
\hline Alcohol & 36 & 0.36 & 2 & 1.39 & .043 \\
\hline Dyspnea & & & & & .012 \\
\hline No dyspnea & 9609 & 95.25 & 131 & 90.97 & \\
\hline Moderate exertion & 449 & 4.45 & 11 & 7.64 & \\
\hline At rest & 30 & 0.30 & 2 & 1.39 & \\
\hline Ventilator dependence & 7 & 0.07 & 1 & 0.69 & .008 \\
\hline COPD & 381 & 3.78 & 8 & 5.56 & .268 \\
\hline Congestive heart failure & 22 & 0.22 & 1 & 0.69 & .231 \\
\hline Hypertension & 4587 & 45.47 & 86 & 59.72 & $<.001$ \\
\hline Ascites & 3 & 0.03 & 0 & 0.00 & .836 \\
\hline Acute renal failure & 2 & 0.02 & 0 & 0.00 & .866 \\
\hline Dialysis dependence & 21 & 0.21 & 0 & 0.00 & .584 \\
\hline Disseminated cancer & 31 & 0.31 & 0 & 0.00 & .505 \\
\hline Wound infection & 46 & 0.46 & 4 & 2.78 & $<.001$ \\
\hline Steroid use & 306 & 3.03 & 10 & 6.94 & .007 \\
\hline Weight loss & 28 & 0.28 & 2 & 1.39 & .014 \\
\hline Bleeding disorders & 111 & 1.10 & 2 & 1.39 & .742 \\
\hline Blood transfusions within $48 \mathrm{~h}$ preoperatively & 38 & 0.38 & 2 & 1.39 & .053 \\
\hline Systemic sepsis & 69 & 0.68 & 2 & 1.39 & .312 \\
\hline Functional status & & & & & .013 \\
\hline Independent & 9864 & 97.78 & 136 & 94.44 & \\
\hline Partially dependent & 170 & 1.69 & 7 & 4.86 & \\
\hline Totally dependent & 12 & 0.12 & 0 & 0.00 & \\
\hline \multicolumn{6}{|c|}{ Laboratory values ${ }^{\mathrm{b}}$} \\
\hline Sodium $(\mathrm{mEq} / \mathrm{L})$ & $139.35 \pm 2.864$ & & $138.97 \pm 3.069$ & & .122 \\
\hline Blood urea nitrogen $(\mathrm{mg} / \mathrm{dL})$ & $15.60 \pm 6.593$ & & $17.08 \pm 8.046$ & & .011 \\
\hline Creatinine $(\mathrm{mg} / \mathrm{dL})$ & $0.90 \pm 0.466$ & & $0.88 \pm 0.285$ & & .639 \\
\hline Albumin $(\mathrm{g} / \mathrm{dL})$ & $4.14 \pm 0.475$ & & $3.93 \pm 0.478$ & & $<.001$ \\
\hline White blood cells $\left(10^{3} \mathrm{c} / \mathrm{mL}\right)$ & $7.27 \pm 2.376$ & & $7.27 \pm 2.541$ & & .996 \\
\hline Hematocrit $(\%)$ & $41.12 \pm 4.458$ & & $38.85 \pm 4.516$ & & $<.001$ \\
\hline Platelets (per mL) & $218.23 \pm 118.158$ & & $226.93 \pm 104.456$ & & .380 \\
\hline INR ratio & $1.00 \pm 0.167$ & & $1.05 \pm 0.326$ & & .003 \\
\hline
\end{tabular}

Abbreviations: COPD, chronic obstructive pulmonary disease; INR, international normalized ratio; UTI, urinary tract infection.

${ }^{a}$ All bolded values represent statistically significant results.

${ }^{\mathrm{b}}$ Values expressed as mean $\pm \mathrm{SD}$. All other values expressed as $\mathrm{N}$ and percent.

${ }^{c}$ Pneumonia and angina were excluded due to $<85 \%$ of patients containing data on preoperative pneumonia or angina status. 
Table 2. Operative variables.

\begin{tabular}{|c|c|c|c|c|c|}
\hline \multirow[b]{2}{*}{ Operative Variables } & \multicolumn{2}{|c|}{ Control } & \multicolumn{2}{|l|}{ UTI } & \multirow[b]{2}{*}{$P$ value $^{\text {a }}$} \\
\hline & No. & $\%$ & No. & $\%$ & \\
\hline Total & 10088 & & 144 & & \\
\hline Days to operation from admission (mean $\pm \mathrm{SD}$ ) & $0.35 \pm 7.909$ & & $0.51 \pm 2.461$ & & .806 \\
\hline Total operating time $($ mean $\pm \mathrm{SD} ; \min )$ & $198.16 \pm 120.054$ & & $250.19 \pm 150.093$ & & $<.001$ \\
\hline Total hospital stay length (mean $\pm \mathrm{SD} ; \mathrm{d})$ & $3.91 \pm 4.856$ & & $8.03 \pm 8.103$ & & $<.001$ \\
\hline Days from operation to death (mean $\pm \mathrm{SD})$ & $7.32 \pm 7.046$ & & 6.00 & & .856 \\
\hline Days from operation to discharge (mean $\pm \mathrm{SD}$ ) & $3.70 \pm 3.367$ & & $7.52 \pm 7.290$ & & $<.001$ \\
\hline Discharge destination & & & & & $<.001$ \\
\hline Home & 6830 & 67.70 & 66 & 45.83 & \\
\hline Other than home & 1111 & 11.01 & 43 & 29.86 & \\
\hline Not reported & 2147 & 21.28 & 35 & 24.31 & \\
\hline Care type & & & & & .243 \\
\hline Inpatient & 9877 & 97.91 & 143 & 99.31 & \\
\hline Outpatient & 211 & 2.09 & 1 & 0.69 & \\
\hline Anesthesia administered & & & & & $<.001$ \\
\hline Epidural & 3 & 0.03 & 0 & 0.00 & \\
\hline General & 10007 & 99.20 & 140 & 97.22 & \\
\hline Local/regional & 2 & 0.02 & 1 & 0.69 & \\
\hline $\mathrm{MAC}^{\mathrm{a}} / \mathrm{IV}$ sedation & 3 & 0.03 & 0 & 0.00 & \\
\hline Spinal & 11 & 0.11 & 3 & 2.08 & \\
\hline Other & 54 & 0.54 & 0 & 0.00 & \\
\hline Wound class & & & & & .840 \\
\hline 1. Clean & 9925 & 98.38 & 141 & 97.92 & \\
\hline 2. Clean/contaminated & 84 & 0.83 & 2 & 1.39 & \\
\hline 3. Contaminated & 20 & 0.20 & 0 & 0.00 & \\
\hline 4. Dirty/infected & 59 & 0.58 & 1 & 0.69 & \\
\hline Total operating time (min) & & & & & .002 \\
\hline$\leq 119$ & 2925 & 28.99 & 23 & 15.97 & \\
\hline$\overline{120} \leq \mathrm{x}<180$ & 2467 & 24.45 & 35 & 24.31 & \\
\hline $180 \leq x<240$ & 1807 & 17.91 & 29 & 20.14 & \\
\hline$\geq 240$ & 2886 & 28.61 & 57 & 39.58 & \\
\hline
\end{tabular}

Abbreviations: MAC, monitored anesthesia care; UTI, urinary tract infection.

${ }^{a}$ All bolded values represent statistically significant values.

SD 13.627; $P<.001)$ and more likely to be female (77.78\% versus $53.37 \% ; P<.001)$. These patients also had significantly higher levels of blood urea nitrogen $(P=.011)$ and greater international normalised ratio $(P=.003)$ but lower levels of serum albumin $(P<.001)$ and hematocrit $(P<$ .001) (Table 1).

There were significant differences in the prevalence of numerous preoperative comorbidities between the 2 cohorts. Patients who experienced UTIs had significantly higher rates of alcohol use $(P=$ $.043)$, dyspnea $(P=.012)$, ventilator dependence $(P$ $=.008)$, hypertension requiring medications $(P<$ $.001)$, open wound/wound infections $(P<.001)$, steroid use for chronic conditions $(P=.007)$, significant weight loss $(P=.014)$, and functional dependence $(P=.013)$. UTI patients also had significantly higher rates of non-insulin-dependent diabetes mellitus but lower rates of insulin-dependent diabetes mellitus $(P<.001)$, as well as smoking history $(P=.007)$ (Table 1$)$.

Patients who developed UTIs following ALIF had significantly longer operative times (250.19 minutes, SD 150.093 versus $198.16 \mathrm{~min}$, SD
120.054; $P<.001$ ), total length of hospital stay (8.03 days, SD 8.103 versus 3.91 days, SD 4.856; $P$ $<.001$ ), and inpatient stay following the operation (7.52 days, SD 7.290 versus 3.70 days, SD 3.367; $P$ $<.001)$. These patients also experienced increased discharge to destinations other than their own homes $(P<.001)$, including separate acute care, skilled facilities, etc. (Table 2).

Upon multivariate analyses, several patient characteristics and comorbidities were found to be significant independent risk factors for postoperative UTIs in patients undergoing ALIF. Female patients were 3.1 times more likely to develop UTIs (95\% confidence interval $[\mathrm{CI}] 2.08-4.64 ; P<.001)$ compared to men, and patients who were at least 60 years of age were 1.5 times more likely to experience postoperative UTIs $(95 \%$ CI $1.07-2.20 ; P=.022)$ than younger patients. Several preoperative comorbidities were found to increase the risk for patients developing UTIs, including significant alcohol use (odds ratio [OR] 6.504, 95\% CI 1.47-28.85; $P=$ .014 ), open wounds or wound infections (OR 3.955, $95 \%$ CI 1.25-12.51; $P=.019)$, and steroid use for chronic conditions (OR 1.965, 95\% CI 1.01-3.82; $P$ 
Table 3. Risk factors for postoperative urinary tract infections.

\begin{tabular}{|c|c|c|c|c|}
\hline \multirow{2}{*}{$\begin{array}{l}\text { Risk Factors } \\
\text { Age }(y)\end{array}$} & \multirow[t]{2}{*}{ Odds Ratio } & \multicolumn{2}{|c|}{ 95\% Confidence Interval } & \multirow[t]{2}{*}{$P$ Value $^{\mathrm{a}}$} \\
\hline & & & & \\
\hline$<60$ & Reference & NA & NA & NA \\
\hline$\geq 60$ & 1.543 & 1.066 & 2.233 & .022 \\
\hline \multicolumn{5}{|l|}{$\operatorname{Sex}$} \\
\hline Male & Reference & NA & NA & NA \\
\hline Female & 3.104 & 2.079 & 4.635 & $<.001$ \\
\hline \multicolumn{5}{|l|}{ Diabetes mellitus } \\
\hline Nondiabetic & Reference & NA & NA & NA \\
\hline NIDDM & 1.685 & 0.902 & 3.148 & .102 \\
\hline IDDM & 1.142 & 0.669 & 1.949 & .626 \\
\hline Smoke & 0.648 & 0.396 & 1.061 & .085 \\
\hline Alcohol use & 6.504 & 1.466 & 28.847 & .014 \\
\hline \multicolumn{5}{|l|}{ Dyspnea } \\
\hline No dyspnea & Reference & NA & NA & NA \\
\hline Moderate exertion & 1.322 & 0.683 & 2.561 & .408 \\
\hline At rest & 3.694 & 0.837 & 16.305 & .084 \\
\hline Ventilator dependence & 3.494 & 0.273 & 44.798 & .336 \\
\hline \multicolumn{5}{|l|}{ Functional status } \\
\hline Independent & Reference & NA & NA & NA \\
\hline Dependent & 1.850 & 0.805 & 4.252 & .147 \\
\hline Hypertension & 1.353 & 0.932 & 1.966 & .112 \\
\hline Open wound/wound infection & 3.955 & 1.251 & 12.506 & .019 \\
\hline Steroid use & 1.965 & 1.011 & 3.82 & .046 \\
\hline Significant weight loss & 3.299 & 0.631 & 17.248 & .157 \\
\hline \multicolumn{5}{|l|}{ Operation duration (min) } \\
\hline$<119$ & Reference & NA & NA & NA \\
\hline$\overline{120} \leq \mathrm{x}<180$ & 1.703 & 0.999 & 2.904 & .050 \\
\hline $180 \leq \mathrm{x}<240$ & 1.895 & 1.085 & 3.311 & .025 \\
\hline$\geq 240$ & 2.337 & 1.431 & 3.818 & .001 \\
\hline
\end{tabular}

Abbreviations: IDDM, insulin-dependent diabetes mellitus; NA, not applicable; NIDDM, non-insulin-dependent diabetes mellitus.

${ }^{a}$ All bolded values represent statistically significant results.

$=.046)$. Longer operative times were also found to independently increase the risk for developing UTIs when compared to operations under 120 minutes, with ORs increasing for each subsequent time stratification: $120 \mathrm{~min} \leq \mathrm{x}<180 \mathrm{~min}(\mathrm{OR} 1.703$, 95\% CI 1.00-2.90; $P=.050), 180 \mathrm{~min} \leq \mathrm{x}<240 \mathrm{~min}$ (OR 1.895, 95\% CI 1.09-3.31; $P=.025), \geq 240 \mathrm{~min}$ (OR 2.337, 95\% CI 1.431-3.82; $P=.001$ ) (Table 3).

Patients who developed UTIs following their procedure also had significantly greater rates of other numerous postoperative complications. A complete list of postoperative complications and $P$ values can be seen in Table 4 . In addition, multivariate logistic regression models demonstrating increased risk for several complications in association with the UTI following ALIF can be found in Table 5.

\section{DISCUSSION}

The current retrospective study sought to identify risk factors that independently increased the risk for postoperative UTIs following ALIF. By identifying independent risk factors for UTI in patients undergoing ALIF, proper prophylactic measures can be taken to decrease the risk of UTI in these patients and prevent associated postoperative adverse events as UTIs have been associated with increased rates of postoperative complications, mortality, and increased length of stay. $3,4,6,17,18$ Indeed, the results of this retrospective study contribute to this growing body of evidence as UTIs were associated with increased postoperative complications following ALIF (Table 5).

Risk factors identified in this study include age $\geq$ 60 years, female sex, alcohol use, chronic steroid use, preoperative open wound or wound infections, and longer operative times as independent risk factors for the development of UTIs following ALIF. To the authors' knowledge, there has been no previously reported association of chronic steroid use with UTI in ALIF. The results of this study support the current literature as the association between chronic steroid use and UTI occurrence has been previously reported in various urological procedures and total joint arthroplasty. ${ }^{19,20}$ Steroids have been known to predispose patients to infectious complications as they are immunosuppressive via anti-inflammatory gene expression and alterations in immune cell trafficking. ${ }^{21,22}$

In addition to chronic steroid use, this current study identifies significant alcohol intake as increas- 
Table 4. Univariate analyses for postoperative complications in patients that developed UTIs.

\begin{tabular}{|c|c|c|c|c|c|}
\hline \multirow[b]{2}{*}{ Postoperative Complications } & \multicolumn{2}{|c|}{ Control } & \multicolumn{2}{|c|}{ UTI } & \multirow[b]{2}{*}{$P$ Value ${ }^{\text {a }}$} \\
\hline & $\mathbf{N}$ & $\%$ & $\mathbf{N}$ & $\%$ & \\
\hline Total & 10088 & & 144 & & \\
\hline Superficial incisional SSI ${ }^{\mathrm{a}}$ & 103 & 1.02 & 8 & 5.56 & $<.001$ \\
\hline Deep incisional SSI & 49 & 0.49 & 3 & 2.08 & .007 \\
\hline Organ/space SSI & 31 & 0.31 & 2 & 1.39 & .023 \\
\hline Wound disruption & 21 & 0.21 & 1 & 0.69 & .211 \\
\hline Pneumonia & 85 & 0.84 & 7 & 4.86 & $<.001$ \\
\hline Unplanned intubation & 31 & 0.31 & 4 & 2.78 & $<.001$ \\
\hline Pulmonary embolism & 56 & 0.56 & 1 & 0.69 & .823 \\
\hline Ventilator dependence $(>48 \mathrm{~h})$ & 44 & 0.44 & 7 & 4.86 & $<.001$ \\
\hline Progressive renal insufficiency & 12 & 0.12 & 0 & 0.00 & .679 \\
\hline Acute renal failure & 10 & 0.10 & 1 & 0.69 & .030 \\
\hline $\mathrm{CVA} /$ stroke & 8 & 0.08 & 1 & 0.69 & .013 \\
\hline Cardiac arrest & 22 & 0.22 & 1 & 0.69 & .231 \\
\hline Myocardial infarction & 30 & 0.30 & 0 & 0.00 & .512 \\
\hline Blood transfusions & 941 & 9.33 & 36 & 25.00 & $<.001$ \\
\hline Deep venous thromboembolism & 80 & 0.79 & 4 & 2.78 & .009 \\
\hline Systemic sepsis & 56 & 0.56 & 12 & 8.33 & $<.001$ \\
\hline Septic shock & 12 & 0.12 & 0 & 0.00 & .679 \\
\hline Death & 21 & 0.21 & 1 & 0.69 & .211 \\
\hline Return to operating room & 321 & 3.18 & 14 & 9.72 & $<.001$ \\
\hline Extended length of stay $(\geq 5 \mathrm{~d})$ & 2351 & 23.30 & 85 & 59.03 & $<.001$ \\
\hline Readmission & 518 & 5.13 & 24 & 16.67 & $<.001$ \\
\hline
\end{tabular}

Abbreviations: CVA, cerebrovascular accident; SSI, surgical site infection.

${ }^{\mathrm{a}} \mathrm{All}$ bolded values represent statistically significant values.

ing the rate of UTI in ALIF patients. Alcohol has long been known to have deleterious effects via various cellular mechanisms when consumed in excess due to subclinical immunosuppressive properties that may only become apparent during physiological stress such as surgery. ${ }^{23,24}$ Future research examining the efficacy of steroid tapering and alcohol cessation in decreasing UTI rates postALIF is warranted.

Female sex and age $\geq 60$ years were also identified as risk factors for precipitating UTI in
ALIF patients. Outside the setting of surgery, females have higher rates of UTIs compared to males when matched for age and other demographic variables due to (1) the shorter female urethra and (2) the proximity of the urethral opening to the end of the gastrointestinal tract. ${ }^{1,25,26}$ Early genotypic studies of Escherichia coli in female UTIs have demonstrated identical genomic profiles to that of enteric E. coli. ${ }^{27}$

Older age is another risk factor that increases the likelihood of developing a UTI following ALIF.

Table 5. Multivariate analyses assessing urinary tract infections as an independent risk factor for experiencing other postoperative complications.

\begin{tabular}{|c|c|c|c|c|}
\hline \multirow{2}{*}{$\begin{array}{l}\text { Postoperative Complications } \\
\text { Superficial incisional SSI }\end{array}$} & \multirow{2}{*}{$\frac{\text { Odds Ratio }}{5.835}$} & \multicolumn{2}{|c|}{ 95\% Confidence Interval } & \multirow{2}{*}{$\begin{array}{r}P \text { Value }^{\mathrm{a}} \\
<.001\end{array}$} \\
\hline & & 2.732 & 12.466 & \\
\hline Deep incisional SSI & 2.846 & 0.825 & 9.822 & .098 \\
\hline Organ/space SSI & 3.763 & 0.804 & 17.603 & .092 \\
\hline Wound disruption & 2.670 & 0.349 & 20.422 & .344 \\
\hline Pneumonia & 5.087 & 2.173 & 11.911 & $<.001$ \\
\hline Unplanned intubation & 6.531 & 2.115 & 20.172 & .001 \\
\hline Pulmonary embolism & 1.219 & 0.166 & 8.969 & .846 \\
\hline Ventilator dependence $(>48 \mathrm{~h})$ & 6.248 & 2.409 & 16.203 & $<.001$ \\
\hline Acute renal failure & 5.041 & 0.562 & 45.235 & .148 \\
\hline $\mathrm{CVA} /$ stroke & 2.334 & 0.168 & 32.503 & .528 \\
\hline Cardiac arrest & 2.649 & 0.342 & 20.491 & .351 \\
\hline Blood transfusions & 2.144 & 1.433 & 3.208 & $<.001$ \\
\hline Deep venous thromboembolism & 2.873 & 0.999 & 8.262 & .050 \\
\hline Systemic sepsis & 14.328 & 7.096 & 28.929 & $<.001$ \\
\hline Death & 2.373 & 0.275 & 20.479 & .432 \\
\hline Return to operating room & 2.548 & 1.424 & 4.558 & .002 \\
\hline Extended length of stay $(\geq 5 \mathrm{~d})$ & 3.631 & 2.557 & 5.156 & $<.001$ \\
\hline Readmission & 3.044 & 1.920 & 4.828 & $<.001$ \\
\hline
\end{tabular}

Abbreviations: CVA, cerebrovascular accident; SSI, surgical site infection.

${ }^{a}$ All bolded values represent statistically significant values. 
Benign prostatic hypertrophy in older men leads to increased urinary stasis that promotes the growth of bacterium in the urinary tract. ${ }^{28}$ Menopausal changes have also been known to predispose older women to UTI development due to decreases in estrogen in the vaginal epithelium. ${ }^{29}$ Additionally, immune function declines as patients age, potentially preventing older individuals from mounting adequate immune responses to prevent UTI. ${ }^{30}$ Bohl et al previously reported that female sex increased the risk for UTI in posterior approaches to lumbar fusion. In addition, patients $\geq 60$ years old had an increased risk of UTI that correlated positively with increasing age, whereas patients ages 50 to 59 years old had no difference in UTI rates compared to a younger population cohort. ${ }^{14}$ As age and sex are nonmodifiable risk factors for UTI, a lower threshold of suspicion for UTI should be held for patients undergoing ALIF who are female and $\geq 60$ years of age. Although appropriate removal of catheters is a standard of care, ${ }^{25}$ stringent adherence to guidelines for these high-risk patients is warranted to prevent further morbidity.

The current retrospective study lends further supporting evidence to a growing body of literature that reports longer operative times lead to increased UTI development. We report operative times greater than 119 minutes demonstrate a direct positive correlation with increased postoperative UTI development - each additional stratification of operative time demonstrated increased statistical significance. Previous reports have shown that longer operative times increase the risk of UTI across all surgical fields, including posterior lumbar fusion and single-level lumbar fusion of different approaches. ${ }^{14,31-33}$ To the authors' knowledge, this relationship has not been previously demonstrated in anterior approaches to the lumbar spine. The ACS-NSQIP database does not report why certain operations are of longer duration or the specific length of operative times, likely representing limitations to this study. Longer operative times can be due to a number of factors including additional levels, excessive bleeding requiring operative control, high soft-tissue body habitus, and surgeon experience or case volume. Longer operative times have been reported to increase the risk of pathogen exposure and increased iatrogenic tissue trauma that may increase the risk of UTI. ${ }^{32}$ Increased operative time can also lead to UTI via hypothermia induced lymphocytic dysfunction. ${ }^{34}$ In addition, an in- creased operative time could also indicate a more complex operation that could require extended postoperative catheterization, allowing bacteria more time to ascend the urinary tract and cause infection. ${ }^{1}$ Further quality improvement focused work investigating methods to reduce operative time in ALIF may prove beneficial in reducing UTI rates.

Our study identified preoperative open wounds or wound infections to be an independent risk factor for the development of UTI in ALIF patients. UTIs have been reported to increase the risk of various infectious complications in the postoperative period following many surgical procedures. Asymptomatic and symptomatic preoperative UTIs and postoperative UTIs have been linked to increased rates of infectious complications, sepsis, morbidity, and mortality. ${ }^{35,36}$ Multivariate analyses in this study provides supporting evidence as UTIs were found to increase the risk for postoperative surgical site infection and pneumonia as well. These complications likely arise from more severe UTIs that can lead to urosepsis, widespread migration of bacteria throughout the body, and subsequent infectious complications. However, to the authors' knowledge, there appears to be no other reports of the inverse relationship.

There is a lack of literature investigating preoperative wounds and their association with UTI in the postoperative period. The exact link between preoperative wounds that lead to UTI in the postoperative period is as of yet unclear. It is plausible that those with preoperative wound infections may already be immunocompromised, leading to an increased likelihood of developing UTIs following ALIF. As the ACS-NSQIP database does not provide specific parameters of immune functioning in these patient files, this represents a limitation of the database for this study. Staphylococcus aureus has been implicated in primary $S$. aureus UTI as the bacterium can seed the urinary tract hematogenously as opposed to fecal-perinealurethral contamination by enteric organisms. ${ }^{37}$ Hematogenous spread of staphylococcal species may very well lead to UTI in ALIF patients. Future work analyzing the correlation of nonenteric bacterium in UTI and the presence of preoperative open wounds or wound infections is warranted. In light of these results, surgeons should postpone elective ALIF operation until all open wounds and wound infections have been resolved to reduce the risk of UTI and subsequent increased morbidity. 
This current retrospective study presents with several limitations. This study only analyzed demographic variables, comorbidities, and complications in which $\geq 85 \%$ of the patient files were recorded. By excluding other variables that did not meet these inclusion criteria, several other demographic factors, postoperative complications, and comorbidities that may in reality be associated with UTIs may have not been reported. In addition, the inability to verify the information being entered into the database is a limitation inherent to all nationwide database studies. Furthermore, CPT codes are not designed for research purposes - the use of CPT codes can introduce coding bias based on financial reimbursement. ${ }^{38}$ This current study included all ALIF procedure files that met inclusion criteria. However, as ALIF is indicated for a variety of spinal conditions, distinct pathologies may represent systematic confounding variables that this retrospective study could not account for. An additional confounding variable not accounted for in this study is that all ALIF procedures, including multilevel operations, were included. Future studies examining the effect of multilevel operations on postoperative UTI rates may prove to demonstrate increasing risk of UTI with increasing levels of operation. Finally, the ACS-NSQIP database does not provide details on catheter use. Specific information regarding duration of catheterization in ALIF patients could provide data on whether there is a particular period of time that UTI risk becomes unacceptable in the postoperative period. Elucidating a specific catheterization duration after which the risk of UTI becomes unacceptable may prove useful in formulating quantitative guidelines in the future.

\section{CONCLUSIONS}

The present study demonstrated that age $\geq 60$ years, female sex, alcohol use, chronic steroid use, preoperative open wound/wound infections, and longer operative times increase the risk for postoperative UTI following ALIF. Identification of risk factors for UTI after ALIF may allow surgical care teams to better risk stratify patients in order to initiate more aggressive prophylactic measures to mitigate the effects of UTIs and their associated complications. The earliest possible removal of catheters must be stringently adhered to in these patients. Providers should have lower thresholds of suspicion for high-risk patients in the postoperative period following ALIF to avoid UTI and its associated complications. Quality improvement initiatives focusing on ensuring earliest postoperative catheter removal may help mitigate risk in these high-risk patients.

\section{REFERENCES}

1. Chenoweth CE, Gould CV, Saint S. Diagnosis, management, and prevention of catheter-associated urinary tract infections. Infect Dis Clin North Am. 2014;28(1):105-119. doi:10.1016/j.idc. 2013.09.002.

2. Ksycki MF, Namias N. Nosocomial urinary tract infection. Surg Clin North Am. 2009;89(2):475-481. doi:10. 1016/j.suc.2008.09.012.

3. Schuller K, Probst J, Hardin J, Bennett K, Martin A. Initial impact of Medicare's nonpayment policy on catheterassociated urinary tract infections by hospital characteristics. Health Policy. 2014;115(2-3):165-171. doi:10.1016/j.healthpol. 2013.11.013.

4. Klevens RM, Edwards JR, Richards CL Jr, et al. Estimating health care-associated infections and deaths in U.S. hospitals, 2002. Public Health Rep. 2007;122(2):160-166. doi: $10.1177 / 003335490712200205$.

5. Shepard J, Hadhazy E, Frederick J, et al. Using electronic medical records to increase the efficiency of catheter-associated urinary tract infection surveillance for National Health and Safety Network reporting. Am J Infect Control. 2014;42(3):e3336. doi:10.1016/j.ajic.2013.12.005.

6. Weisensel NA, Olson TJP, Rajamanickam V, Kennedy GD. Urinary tract infection: tip of a postoperative complication iceberg? J Surg Res. 2013;179(2):287. doi:10.1016/j.jss.2012.10. 564.

7. Saint S, Meddings JA, Calfee D, Kowalski CP, Krein SL. Catheter-associated urinary tract infection and the Medicare rule changes. Ann Intern Med. 2009;150(12):877-884. doi:10. 7326/0003-4819-150-12-200906160-00013.

8. Capener N. Spondylolisthesis. Br J Surg. 1932;19(75):374 386. doi: 10.1002/bjs. 1800197505 .

9. Ito H, Tsuchiya J, Asami G. A new radical operation for Pott's disease. J Bone Joint Surg. 1934;16(3):499-515.

10. Mobbs RJ, Loganathan A, Yeung V, Rao PJ. Indications for anterior lumbar interbody fusion. Orthop Surg. 2013;5(3):153-163. doi:10.1111/os.12048.

11. Min JH, Jang JS, Lee SH. Comparison of anterior- and posterior-approach instrumented lumbar interbody fusion for spondylolisthesis. J Neurosurg Spine. 2007;7(1):21-26. doi:10. 3171/SPI-07/07/021.

12. Than KD, Wang AC, Rahman SU, et al. Complication avoidance and management in anterior lumbar interbody fusion. Neurosurg Focus. 2011;31(4):E6. doi:10.3171/2011.7. FOCUS11141.

13. Scaduto AA, Gamradt SC, Yu WD, Huang J, Delamarter RB, Wang JC. Perioperative complications of threaded cylindrical lumbar interbody fusion devices: anterior versus posterior approach. J Spinal Disord Tech. 2003;16(6):502-507.

14. Bohl DD, Ahn J, Tabaraee E, et al. Urinary tract infection following posterior lumbar fusion procedures: an American College of Surgeons National Surgical Quality 
Improvement Program study. Spine (Phila Pa 1976). 2015;40(22):1785-1791. doi:10.1097/BRS.0000000000001003.

15. American College of Surgeons National Surgical Quality Improvement Program. User Guide for the 2016 ACS NSQIP Participant Use Data File (PUF). October 2017. https://www. facs.org/ /media/files/quality\%20programs/nsqip/nsqip_puf_ userguide_2016.ashx. Accessed May 2018.

16. American College of Surgeons National Surgical Quality Improvement Program. User Guide for the 2012 ACS NSQIP Participant Use Data File (PUF). October 2013. https://www. facs.org/ /media/files/quality\%20programs/nsqip/ug12.ash. Accessed May 2018.

17. Saint S. Clinical and economic consequences of nosocomial catheter-related bacteriuria. Am J Infect Control. 2000;28(1):68-75.

18. Platt R, Polk BF, Murdock B, Rosner B. Mortality associated with nosocomial urinary-tract infection. $N$ Engl J Med. 1982;307:637-642. doi:10.1056/NEJM198209093071101.

19. Vigil HR, Hickling DR. Urinary tract infection in the neurogenic bladder. Transl Androl Urol. 2016;5(1):72-87. doi:10.3978/j.issn.2223-4683.2016.01.06.

20. Alvarez AP, Demzik AL, Alvi HM, Hardt KD, Manning DW. Risk factors for postoperative urinary tract infections in patients undergoing total joint arthroplasty. Adv Orthop. 2016;7268985. doi:10.1155/2016/7268985.

21. Klein $\mathrm{NC}$, Go $\mathrm{CH}$, Cunha BA. Infections associated with steroid use. Infect Dis Clin North Am. 2001;15(2):423-432.

22. Coutinho AE, Chapman KE. The anti-inflammatory and immunosuppressive effects of glucocorticoids, recent developments and mechanistic insights. Mol Cell Endocrinol. 2011;335(1):2-13. doi:10.1016/j.mce.2010.04.005.

23. Szabo G, Saha B. Alcohol's effect on host defense. Alcohol Res. 2015;37(2):159-170.

24. Latvala J, Parkkila S, Niemela O. Excess alcohol consumption is common in patients with cytopenia: studies in blood and bone marrow cells. Alcohol Clin Exp Res. 2004;28(4):619-624.

25. Hooton TM, Bradley SF, Cardenas DD, et al. Diagnosis, prevention, and treatment of catheter-associated urinary tract infection in adults: 2009 International Clinical Practice Guidelines from the Infectious Diseases Society of America. Clin Infect Dis. 2010;50(5):625-63. doi:10.1086/650482.

26. Flores-Mireles AL, Walker JN, Caparon M, Hultgren SJ. Urinary tract infections: epidemiology, mechanisms of infection and treatment options. Nat Rev Microbiol. 13(5):269-284. doi:10.1038/nrmicro3432.

27. Yamamoto S, Tsukamoto $\mathrm{T}$, Terai A, Kurazono $\mathrm{H}$, Takeda Y, Yoshida O. Genetic evidence supporting the fecalperineal-urethral hypothesis in cystitis caused by Escherichia coli. J Urol. 1997;157(3):1127-1129.

28. Rowe TA, Juthani-Mehta M. Urinary tract infection in older adults. Aging Health. 2013;9(5):10.2217/ahe.13.38. doi: 10.2217/ahe.13.38.

29. Minardi D, d'Anzeo G, Cantoro D, Conti A, Muzzonigro G. Urinary tract infections in women: etiology and treatment options. Int J Gen Med. 2011;4:333-343. doi:10. 2147/IJGM.S11767.

30. Montecino-Rodriguez E, Berent-Maoz B, Dorshkind K. Causes, consequences, and reversal of immune system aging. $J$ Clin Invest. 2013;123(3):958-965. doi:10.1172/JCI64096.

31. Qin C, de Oliveira G, Hackett N, Kim JY. Surgical duration and risk of urinary tract infection: an analysis of 1 , 452, 369 patients using the National Surgical Quality Improvement Program (NSQIP). Int J Surg. 2015;20:107-112. doi:10. 1016/j.ijsu.2015.05.051.

32. Procter LD, Davenport DL, Bernard AC, Zwischenberger JB. General surgical operative duration is associated with increased risk-adjusted infectious complication rates and length of hospital stay. J Am Coll Surg. 2010;210(1):60-65.e1-2. doi:10.1016/j.jamcollsurg.2009.09.034.

33. Kim BD, Hsu WK, De Oliveira GS Jr, Saha S, Kim JY. Operative duration as an independent risk factor for postoperative complications in single-level lumbar fusion: an analysis of 4588 surgical cases. Spine (Phila Pa 1976). 2014;39(6):51020. doi:10.1097/BRS.0000000000000163.

34. Beilin B, Shavit Y, Razumovsky J, Wolloch Y, Zeidel A, Bessler H. Effects of mild perioperative hypothermia on cellular immune responses. Anesthesiology. 1998;89(5):1133-1140.

35. Pokrzywa CJ, Papageorge CM, Kennedy GD. Preoperative urinary tract infection increases postoperative morbidity. J Surg Res. 2016;205(1):213-220. doi:10.1016/j.jss.2016.06.025.

36. Nunez-Pereira S, Rodriguez-Pardo D, Pellise F, et al. Postoperative urinary tract infection and surgical site infection in instrumented spinal surgery: is there a link? Clin Microbiol Infect. 2014;20(8):768-773. doi:10.1111/1469-0691.12527.

37. Baraboutis IG, Tsagalou EP, Lepinski JL, et al. Primary Staphylococcus aureus urinary tract infection: the role of undetected hematogenous seeding of the urinary tract. Eur $J$ Clin Microbiol Infect Dis. 2010;29(9):1095-1101. doi:10.1007/ s10096-010-0967-2.

38. Yoshihara H, Yoneoka D. Understanding the statistics and limitations of large database analyses. Spine (Phila Pa 1976). 2014;39(16):1311-1312. doi:10.1097/BRS.0000000000000352.

Disclosures and COI: There are no acknowledgements or financial disclosures to be made. This study was exempt from Institutional Review Board approval.

Corresponding Author: Danny Lee, MD, 2300 I St NW, Washington, DC 20052. Phone: (201) 921-0659; Email: dannylee@gwu.edu.

Published 28 August 2020

This manuscript is generously published free of charge by ISASS, the International Society for the Advancement of Spine Surgery. Copyright (c) 2020 ISASS. To see more or order reprints or permissions, see http://ijssurgery.com. 